\title{
ILCEA
}

Revue de l'Institut des langues et cultures

d'Europe, Amérique, Afrique, Asie et Australie

22 | 2015

La révolution théâtrale dans le Río de la Plata

\section{Valores sociolingüísticos e interculturales en la recepción de La omisión de la Familia Coleman de Claudio Tolcachir}

Sociolinguistic and Intercultural Values in Receiving La omisión de la Familia Coleman by Claudio Tolcachir

Valeurs sociolinguistiques et interculturelles dans la réception de La omisión de la Familia Coleman de Claudio Tolcachir

\section{Marcelino Cotilla Vaca}

\section{OpenEdition}

\section{Journals}

Edición electrónica

URL: http://journals.openedition.org/ilcea/3220

DOI: $10.4000 /$ ilcea.3220

ISSN: 2101-0609

Editor

UGA Éditions/Université Grenoble Alpes

Edición impresa

ISBN: 978-2-84310-295-0

ISSN: $1639-6073$

Referencia electrónica

Marcelino Cotilla Vaca, « Valores sociolingüísticos e interculturales en la recepción de La omisión de la Familia Coleman de Claudio Tolcachir », ILCEA [En línea], 22 | 2015, Publicado el 01 marzo 2015, consultado el 19 abril 2019. URL : http://journals.openedition.org/ilcea/3220 ; DOI : 10.4000/ ilcea.3220

Este documento fue generado automáticamente el 19 abril 2019.

(C) ILCEA 


\title{
Valores sociolingüísticos e
} interculturales en la recepción de La omisión de la Familia Coleman de Claudio Tolcachir

\author{
Sociolinguistic and Intercultural Values in Receiving La omisión de la Familia \\ Coleman by Claudio Tolcachir \\ Valeurs sociolinguistiques et interculturelles dans la réception de La omisión \\ de la Familia Coleman de Claudio Tolcachir
}

Marcelino Cotilla Vaca

1 Con estas líneas pretendo recalar unos instantes en el estudio del teatro de Claudio Tolcachir (Buenos Aires, 1975). Para ello quisiéramos partir de una representación concreta, con unas coordenadas espacio-temporales precisas, de su obra más aplaudida por todos los teatros del mundo, La omisión de la Familia Coleman (2005). Esas coordenadas se corresponden con el Teatro Piccolo de Milán, la noche del 17 de abril de 2012.

2 Este planteamiento no pretende ser más (ni menos) que una posición desde un punto de vista del espectador, del espectador del acontecimiento no sólo poiético sino convivial, junto a una serie de espectadores actantes de un acontecimiento teatral sin posible repetición por cuanto actualización única y concreta de una serie de posibilidades siempre implícitas en la propuesta escénica.

3 En el Piccolo de Milán se dio cita un público heterogéneo, no sólo nativos de códigos lingüísticos distintos, sino que por ende y sobre todo, pertenecían a tradiciones literarias, dramáticas y socio-culturales diferentes. En concreto me refiero a numerosos estudiantes de español lengua extranjera de niveles medios y altos (B1.2 en adelante), de origen italiano, junto a italianos con escaso conocimiento de la lengua española que leían la traducción con subtítulos. Junto a ellos un nutrido grupo de compañeros de la comunidad educativa en lengua española, docentes sobre todo de origen español que en muchos casos conocen sólo a muy grandes rasgos la cultura argentina, sin olvidar justamente al 
grupo de argentinos, no sólo actores, sino también público, aunque minoritario en este caso.

4 Esta heterogeneidad cultural nos indujo a considerar un posible estudio de recepción, a través de las reacciones del público y de entrevistas personales que nos ayudaran a confirmar o a desmentir en una u otra medida nuestras iniciales impresiones. Lo que nos atrajo de la situación es que el sistema cultural argentino, de un determinado estado del sistema cultural argentino en el que se inscribía el acontecimiento teatral, se situara de por sí ante una situación compleja, ofreciéndose codificado ante otras dos situaciones culturales con las que por historia, como todos sabemos, comparte afinidades, y frente a las que plantea diferencias a veces no tan sutiles como en un primer momento se pudiera llegar a pensar.

5 Cuando hacemos el inmenso esfuerzo de no caer en banalidades al referirnos al término cultura, no pretendemos quedarnos en lo que a veces se entiende por aspectos culturales de la lengua, elemento que restringiría excesivamente el estudio de una obra teatral, de cualquier obra teatral, pero desde luego de ésta en donde las implicaciones, y las implicaturas, abarcan muchos aspectos. Cuando aludo a esquema cultural me refiero a lo que Clifford Geertz llama "esquema históricamente transmitido de significaciones representadas en símbolos (objeto, acto, hecho, palabra, gesto), un sistema de concepciones heredadas y en forma simbólica a través de medios por los que los hombres comunican, perpetúan y desarrollan su conocimiento y sus actitudes frente a la vida" (Geertz, 2000: 27). Por ejemplo, a través de un "acontecimiento teatral", concepto que amplía y multiplica la perspectiva del concepto "texto teatral".

6 La obra a la que remite ese acontecimiento en cuestión, y de la que ya me he ocupado en algún otro momento (Cotilla Vaca, 2013), es La omisión de la familia Coleman, obra de la Postdictadura cultural argentina. Dubatti considera el teatro argentino de la Postdictadura "teatro de los muertos" (Dubatti, 2014). Para este investigador, "la Postdictadura remite a una unidad por su cohesión profunda en el redescubrimiento y la redefinición del país bajo las consecuencias de la dictadura. Una vasta zona del teatro actual trabaja sin pausa, y de diferentes maneras, en la asunción del horror histórico, la construcción de memorias del pasado, la denuncia y el alerta de lo que sigue vivo de la dictadura en el presente" ("Por qué hablamos") (Dubatti, 2008).

7 Por su parte Macarena Trigo (2010) describe el quehacer de Tolcachir como "una poética de lo roto". Y lo roto emerge en la escena de Tolcachir de los muertos, sí, aunque quizás no sólo. Así, por ejemplo, el "desaparecido" no es un "muerto" y, como sabemos, en esta diferencia se encuentra el trauma de buena parte de tres generaciones de argentinos, pero la ausencia, además, va más allá del concepto de "desaparecido" y tiene mayores implicaciones dramáticas. Por lo que se refiere a Tolcachir, además, el personaje ausente que tantas veces signa el acontecer de los presentes, no es necesariamente ni un muerto ni un desaparecido (no en la acepción histórica restringida del término "desaparecido"). El mismo autor lo confirma:

A mí siempre me interesaron las ausencias que tienen más fuerza dramática que una presencia, porque lo ausente no tiene solución y vive dentro nuestro como una pregunta eterna. Uno no puede enfrentarse con quien no está. Mis personajes por lo general no tienen la facilidad de resolver sus problemas, y una historia viva, pero sin solución por ausencia, es un peso muy grande para seres tan incapaces como los míos (Cotilla Vaca, 2013). 
No pretendemos ahondar, sin embargo, en encuadres históricos. No es nuestro objetivo aquí. Sí que queremos, por un lado, constatar que dentro de estos parámetros, la obra de Tolcachir se erige como un manifiesto perfectamente verosímil de esa sociedad que representa, que en ello reside gran parte de su eficacia como ente ontológicamente vivo y en plena evolución psicológico-social. Por otro lado, nos gustaría partir de las ideas reiteradas en otras ocasiones por el profesor Dubatti (2011: 17), quien insiste en que el estudio de la obra teatral no debe restringirse bajo perspectivas de ente comunicativo o semiótico, puesto que la obra dramática no necesariamente comunica ni significa. Creemos que tal cuestión implica un punto de inflexión en la evolución de los estudios teatrales, más en los estudios de la obra dramática argentina contemporánea, desde la que habla Dubatti. De hecho, el acontecimiento teatral ve cómo distintos individuos que comparten un espacio presencial y simultáneo reaccionan de muy diferentes formas.

9 Tomamos de nuevo conceptos de la filosofía del teatro que son del profesor Dubatti (2010: 61) y partimos de ese "acontecimiento convivial" que, en nuestra opinión en una situación inenarrable por cuantas implicaturas personales y sociales se desarrollaron, reunió a una serie de técnicos, actores, personajes de ficción y público, con códigos lingüísticos, paralingüísticos y socio-culturales distintos, aunque a veces superpuestos. De todo ello quisiéramos extraer algunas consideraciones que no pueden ser más que incompletas pero que pretenden ser al menos orientativas.

10 Está claro, pese al intento, que quien observa a tales individuos desde el conocimiento previo pero obviamente siempre inconcluso de las culturas a las que pertenecen, cree interpretar reacciones desde un punto de vista pretendidamente distante, o quizás sólo en cierto modo distante, con la curiosa paradoja de que se trata de un individuo que participa del convivio y en cierto modo de las tres culturas. La posición privilegiada que nos puedan permitir tales conocimientos se vuelve asimismo un revulsivo y una dificultad para que estas notas puedan ser del todo válidas por cuanto que adolecen de la falta de esa pretendida objetividad. No obstante, quieren servir no más, aunque tampoco menos, como intento de acercamiento metodológico para comprender el desarrollo de una recepción concreta de dicha obra teatral.

11 Para un análisis de las reacciones y evoluciones del "acontecimiento de expectación" (Dubatti 2010: 64) tal vez deberíamos detenernos antes en los estudios hispánicos sobre la pragmática sociocultural, centrados en el amplio espectro que abarca el concepto de cortesía, tanto verbal como no. Los estudios sobre la cortesía pueden ayudarnos a comprender algunas de las diferencias que se establecen no sólo entre el público hispano y no hispano (italiano, para mayor concreción), sino incluso entre los actantes del acontecimiento poiético de origen argentino y el público español, que constituía al menos un tercio de la sala de butacas. Para ello creo que resulta muy útil referirse a las investigaciones del grupo de trabajo EDICE de la Universidad de Estocolmo, dirigido por la profesora Diana Bravo. La profesora Bravo define la estrategia de cortesía como "una acción que consiste en hacer pasar un acto $a$ por un acto $b$ con la finalidad de quedar bien con el otro" (2005: 31-32). Podemos hacer un paréntesis en esta exposición para recordar lo que de autoría colectiva tienen las representaciones de Tolcachir, no sólo en lo que se refiere a la puesta en escena, sino incluso a la propia conformación del texto y que el mismo autor no se cansa de reiterar una y otra vez. La historia del proceso creativo de Tolcachir, que se lanza a las tablas para ensayar sin el texto en la mano, alarga las implicaciones autoriales del proceso poiético y complica las relaciones autor-personajeauditorio, puesto que en cierto modo un mismo actante puede cubrir esas tres facetas, 
como lo demuestra el hecho de que la misma Macarena Trigo, que era actriz en la representación, había colaborado en el proceso creativo de la obra, en el texto (antes de ser escrito) y en la conformación de gestos y actitudes.

Volviendo a lo que nos ocupa, como lo asegura la profesora Bravo, "la cortesía, considerada como un fenómeno sociocultural, se confirma, se actualiza, se modifica o se revierte en la situación de interlocución real" (2004:8) -en nuestro caso habría que hablar del espectador real, quien responde con reacciones distintas a las de otro espectador real. Los parámetros de cortesía o descortesía desde el punto amplio de la pragmática sociocultural, y justamente por la heterogeneidad cultural a la que me he referido, se cruzan así en el desarrollo de un ente más complejo incluso de lo naturalmente complejo ya, por tratarse de un ente teatral mixto de poíesis y representación.

De estas razones nace un primer problema: la culturización de un ente teatral mixto, a partir de implicaciones grupales e individuales bien distintas, que lleva al amplio grupo de espectadores a comportarse desde su individualidad de un modo distinto a cómo podrían haberlo hecho si esa cultura que se desarrolla en escena no se hubiera visto mezclada con la de ellos. El "acontecimiento de expectación" evoluciona alterado, retroalimentándose a sí mismo, gracias a la puesta en marcha de una serie de reacciones en el espectador concreto debidas, no sólo a su individualidad, sino a las reacciones de un grupo heterogéneo de espectadores, entre los que nos incluimos.

Para un análisis de algunos ejemplos que ilustren esta teoría he preferido entresacar algunos momentos de La omisión de la Familia Coleman. Así, por ejemplo, en un determinado momento, Marito insiste en no querer quitarse el pijama para que Gabi pueda lavárselo, y visto que además sería una situación cuando menos no habitual, Gabi se siente contrariada y así lo manifiesta, con gestos bruscos, mientras que la abuela, menos alarmada, resulta más impositiva dentro de su entonación y actitud más tranquilas. Marito agita la cabeza, se cruza de brazos y declara: "Va a ser imposible, abuela" (Tolcachir, 2011:107). Esta actitud incide de modo desigual en el público de la sala.

En dicha actitud se rastrea una rebeldía que para un argentino va más allá de la mera falta de educación. No estaría de más aludir a las palabras de Mempo Giardinelli cuando asegura que

El argentino, desde siempre, arrastra la carga frustrante de la lucha entre la libertad y la censura, entre el permiso y el veto, hecho generador de una gran autoestima, casi proverbial, que algunos pueblos critican en nosotros y que podría explicarse a partir de los sueños de grandeza acuñados durante muchas décadas y generaciones (1998: 37-38).

16 El actor que encarna a Marito, Lautaro Perotti, señalaba justamente al finalizar la obra que en ocasiones como ésta el plantel de actores se sorprendía de que, sin ellos haberlo pretendido, el texto y las actitudes kinésicas hubieran provocado la risa entre el público. E insistimos en el que hecho de que fuera "entre" el público, ya que no se trató de una actitud generalizada. Marito, en realidad, y desde un punto de vista sin duda histriónico (histrionismo que atañe, insisto, al personaje como conformación), lo que está haciendo es reclamar esa libertad, ese sueño de autoafirmación de un personaje desvalido. Tal histrionismo de gestos y palabras, y la entonación exagerada y cortante, pueden mover en un público argentino a la sonrisa. En el Piccolo de Milán se escucharon carcajadas, algo 
bien distinto, y eso es lo que llamaba la atención de los mismos actores, aunque no era la primera vez que sucedía en su infatigable gira.

En 2009, Elena Cancelli presentó en la sede de Brescia de la Università Cattolica una memoria de licenciatura aún sin publicar, Strategie di cortesia e contesti socio-culturali: Spagna e Argentina a confronto. Siguiendo a la profesora Diana Bravo, Cancelli toma como base la categoría de la afiliación como rasgo específico de cortesía en una cultura e identifica la cortesía en la identidad cultural argentina a través de fuertes redes de solidaridad. Y ésta es la palabra clave: la solidaridad. Ello contrasta, siempre según esta autora, con rasgos individualistas de los últimos tiempos, individualismo nada extraño a la obra, que es lo que conduce a la alienación y que llevará a la desesperanza en la escena final en la que Marito enfermo es abandonado dentro de la casa a su propia suerte.

El desarraigo histórico ha dado paso, como el mismo Claudio Tolcachir nos lo confesaba en una entrevista personal, a un desarraigo individual e íntimo. Desde "esa historia de un país en el que yo me crié", dice el autor, "en donde hay miles de personas que no sabemos dónde están", pero también en un país en donde "existen historias de gente que abandona sus hogares y sus familias. Y desaparecen de sus responsabilidades y también es una tragedia contemporánea el no poder asumir (a) tu familia. Sucede mucho. Y me duelen profundamente esas historias" (Cotilla Vaca, 2013).

Si los personajes de La omisión son justamente personajes signados por un desarraigo personal, un abandono, no es de extrañar que en esa descortesía por insolidaridad se esté produciendo una rebeldía, una actitud consciente de no querer colaborar, con fuerza, con virulencia. De ahí el cruce de brazos de Marito y su "eso es imposible".

Ahora bien, como lo recuerda Cancelli, a diferencia de la sociedad argentina, la sociedad española, a la que pertenecía un tercio del público de la sala, no basa sus coordenadas de cortesía en la idea de solidaridad, sino en la de "confianza", algo opuesto en cierto modo, como luego veremos, a la idea de cortesía del país en donde se representó la obra. La confianza lleva incluso a confesar la bajeza ante los otros y el rechazo a lavarse en público como un principio vital (situación contradictoria pero no impensable) a través de palabras y gestos exagerados, y eso es lo que empuja al humor para el público español. No me atrevería a decir que se trata de un déficit de profundización en el personaje, sino más bien, y aquí es donde salta la grandeza del acontecimiento convivial, de una nueva apertura, de una plurisignificación quizás incluso no pretendida por el autor o los autores que gestaron la actitud de Marito.

21 Por otro lado, la idea de "cortesía" en Italia pasa por el concepto de distancia social, en donde subyace la idea de una mayor privacidad y autonomía. Los gestos y las palabras se incluyen siempre en la evasión de la negatividad. De hecho, las buenas maneras imponen que el "no" explícito o de declaraciones de principio como las de Marito se eviten lo más posible en las respuestas.

22 La profesora Frescura (1997) analiza la situación oferta-rechazo en italiano -que no se corresponde con este momento, pero pienso que puede servirnos para comprender la reacción del público. Frescura llega a la conclusión de que las negaciones explícitas (o negaciones reforzadas de principio como la de Marito) no constituyen más de un $5 \%$ en lengua italiana. Por el contrario, otras estrategias, como la del rechazo táctico o el conclusivo son casi exclusivas y suman más del $90 \%$. Todo es más tácito. En los gestos la negación social pasa por el rostro, no siempre por las palabras, y mucho menos por el resto del cuerpo. De ahí que una salida de tono como la de Marito ("eso es imposible") un 
italiano la juzgue como excesiva, de clara intención humorística, pero no desencadenante de la carcajada, justamente por el exceso que atentaría contra la verosimilitud (insisto, siempre según el público italiano) mientras que el español, que considera que la "confianza" se respeta y por tanto la verosimilitud también, más tratándose de un ámbito familiar, encuentra un desacomodo entre la intención (negar sin más) y el histrionismo, lo que le lleva a la carcajada.

Por todo ello, y paradójicamente, tal vez podamos afirmar que la similar actitud entre un público argentino y uno italiano (la sonrisa molesta frente a la carcajada del español) se encuentren paradójicamente más alejadas entre sí por motivación que la de ambos frente a la del español. Las reacciones de un personaje tan rico como Marito plantean también desacomodos en la recepción de la ironía de la solidaridad que él mismo pone en solfa antes de que el resto de los personajes las pongan en práctica contra él mismo a lo largo de la obra, con apoteosis en el final. Para un argentino podría ser una ironía de la solidaridad: se es aparentemente solidario porque aparentemente uno se preocupa por el otro; para un italiano, tal vez se trate de un exceso (exceso de arrogancia: el otro es tratado como mísero, opuesto extremo del tratamiento como señor), que no justificaría la carcajada, sino incluso el rechazo; para un español, por el contrario, la percepción sería la de un defecto, demasiado sutil, poco excesivo (no hay un explícito ataque a la confianza), lo que tampoco justificaría la carcajada. Así, por ejemplo, según Macarena Trigo,

[...] el acuse de recibo de Marito siempre es el mismo: Ah, algo difícil de interpretar como afirmación y que, por momentos, más allá de identificarlo como una muletilla de su modo de hablar, nos vemos tentados a juzgar como una tomadura de pelo, un chiste del que sólo él puede reírse. Antes de cada uno de esos escuetos $A h$, existe todo un hilo de pensamiento que no podemos reconstruir y sobre ese vacío se levanta la lógica interna de un personaje que fascina por ser del todo imprevisible (2010).

Es decir, hay presupuestos pragmáticos, modificables en cada situación en que Marito proclama su "Ah". Como lo indica el profesor Briz Gómez, "la evaluación de la cortesía verbal sólo es posible en el proceso de interacción mismo" (2004: 79). En el caso del "Ah" de Marito, los valores resultan tan dispares que la cortesía y su ruptura componen una amplia y compleja graduatoria. Si partimos de un significado no marcado de sorpresa, que no aparece o no aparece como fundamental y que, usado para objetivos distintos, se carga de una fuerza expresiva potentísima:

1. Deslegitimar las palabras de otro:

Memé: ¡Ay, Marito, qué asco! ¿Te imaginás? La abuelita muerta, arriba del sillón, toda pudriéndose, echando humito... Andá a hacer el desayuno.

Marito: ¿Por?

Memé: Andá a hacer el desayuno y punto.

Marito: ¿Por qué yo?

Memé: Porque lo digo yo.

Marito: Ah. Gabi tiene poca carne, va a tardar poco también.

2. Burla (aunque es otra forma de deslegitimar):

Memé: Ah. Mario, me fijé, y somos grandes. No hay ningún ratón muerto en el cajón.

Sale.

Marito: Ah.

3. Expresión descortés del gusto personal (aunque la burla está también implícita):

Marito: (A la abuela) Abuela, ¿él quién es?

Abuela: El remisero de Verónica.

Marito: ¿Y cómo se llama? 
Abuela: Hernán.

Marito: Ah.

Podríamos alegar muchos más ejemplos de disimetría en las reacciones del público, pero para no alargar excesivamente nuestra intervención pondré sólo uno. Partimos de dos sociedades en donde la desigualdad social alcanza cotas medias (como la italiana) o altas (como la argentina), según las tablas aportadas por el coeficiente de Corrado Gini (2009): la igualdad utópica sería 1; la desigualdad total, 0 . Italia se situaría en torno a un 0,37, Argentina por encima del 0,50. El sistemático ataque por parte de los hermanos a los principios comportamentales del personaje de Verónica posee repercusiones muy interesantes para nuestro estudio. Verónica es la hermana de la familia que el padre de los Coleman ha preferido beneficiar, ella se avergüenza de su familia, de sus hermanos, por supuesto de su madre, porque es una rama familiar que pertenece a una clase social más baja. Ese ataque por parte del resto de los hermanos posee efectos humorísticos indudables y que mueven a la carcajada ante situaciones extremas. Los gestos de Verónica, su modo de vestir y de reaccionar no escapan a este ámbito. Para una sociedad de menor desigualdad social como la España de 2012 (que se sitúa por debajo del 0,35 según el coeficiente de Gini) estas actitudes no llevan a la carcajada, puesto que no las identifica en la sociedad actual y parecen sin más actitudes y gestos exagerados. Además, se sentirían como reprobables por parte del conjunto de la sociedad, incluso por parte del colectivo social que la propia Verónica representa. Creemos que esto habría provocado que en el Teatro Piccolo de Milán se escucharan carcajadas generalizadas entre el público italiano, con un público español que se sonreía por intentar acompañar la hilaridad generalizada, pero que podía sentir exagerada sin más e incluso poco verosímil la actitud de Verónica.

Estas consideraciones, sin duda sólo orientativas, quizás puedan servir de reflexión y quizás, sólo quizás, animar a estudios más profundos en este sentido en la obra de Tolcachir, en sus problemas de recepción, y tal vez, sólo tal vez, al resto del teatro argentino contemporáneo, verdadero bastión de innovación dramática y literaria contemporánea. Habría que profundizar en estudios más allá de las reacciones de hilaridad, cuando hay reacciones de incomodidad, inquietud en el público. Esto requeriría una serie de acercamientos distintos a los estudios de recepción, algo que estas líneas sólo querían señalar como necesidad.

\section{BIBLIOGRAFÍA}

BRAVo Diana (2004), "Panorámica breve acerca del marco teórico y metodológico”, D. Bravo y

A. Briz, Pragmática sociocultural: estudios sobre el discurso de la cortesía en español (pp. 4-10), Barcelona: Ariel.

BRAVo Diana (2005), “Categorías, tipologías y aplicaciones. Hacia una redefinición de la cortesía comunicativa", Estudios de la (des)cortesía en español. Categorías conceptuales y aplicaciones a corpora orales y escritos (pp. 21-52), Buenos Aires: Dunkan. 
CANCELLI Elena (2009), Strategie di cortesia e contesti socio-culturali: Spagna e Argentina a confronto

(Tesi di laurea inédita), Università Cattolica, sede di Brescia.

CORRADO Gini (2009), World Map Gini coefficient, <http://commons.wikimedia.org/wiki/

File:World_Map_Gini_coefficient.png>.

Cotilla VACA Marcelino (2013), “Omisión de palabra y otras rupturas en el teatro de Claudio

Tolcachir”, Actas del XXXIX Congreso del Instituto Internacional de Literatura Iberoamericana,

Universidad de Cádiz, en prensa.

DuBATti Jorge (2008), “Por qué hablamos de Postdictadura 1983-2008”, La revista del CCC, 4, < www.centrocultural.coop/revista/articulo/85/>.

Dubatti Jorge (2010), “Filosofía del Teatro: fundamentos y corolarios”, Gestos. 25 años de teoría y práctica del teatro, 50, 53-81.

DuBATTI Jorge (2011), Introducción a los estudios teatrales, México: Libros de Godot.

DuBATTI Jorge (2014), Filosofía del teatro III. El teatro de los muertos, Buenos Aires: Atuel.

FRESCURA Marina (1997), "Strategie di rifiuto in italiano: uno studio etnografico”, Italica, 74(4),

Linguistics and Pedagogy, 542-559.

GEERTZ Clifford (2000), La interpretación de la cultura, Barcelona: Gedisa.

Teatro Piccolo de Milán (2012), <http://promo.piccoloteatro.org/i/it/spettacoli/2011-2012/ilcaso-della-famiglia-coleman/il-caso-della-famiglia-coleman.html>.

TolCACHIR Claudio (2011), "La omisión de la Familia Coleman", C. Tolcachir, El viento en un violín y otros textos (pp. 101-189), Buenos Aires: Atuel. (Obra estrenada en 2005 en Buenos Aires.)

TRIGo Macarena (2010), “La omisión de la Familia Coleman: una poética de lo roto”, < www.laomisiondelafamiliacoleman.blogspot.it/>.

\section{RESÚMENES}

Nos preguntamos de nuevo por el éxito mundial del teatro de Claudio Tolcachir, sobre todo de La omisión de la familia Coleman, una obra multipremiada, además de representada más de 1500 veces en sólo cinco años en multitud de teatros de todo el mundo.

Para introducirnos en las posibles concausas de este fenómeno, estudiamos sistemáticamente la ruptura reiterada de los principios de cortesía verbal sugeridos por la sociolingüística, también considerando la variación de tales principios en cada una de las sociedades que reciben el acontecimiento teatral. Resulta muy oportuno aquí el concepto de "acontecimiento teatral" del profesor Dubatti, en cuanto en concreto una obra como La omisión..., por la vía de la recepción, se convierte en un ser ontológicamente vivo dada su moldeabilidad social.

Consideramos el profundo enraizamiento en los problemas concretos de personajes únicos y vinculados a una sociedad contemporánea perfectamente reconocible, en sus angustias, sus carencias, sus frustaciones y, en tantas ocasiones, sus acciones o inacciones coherentemente verídicas. De esta fuerza realista, pero también del hiperrealismo, surgen comportamientos y expresiones lingüísticas, pragmalingüísticas y paralingüísticas, que definen nítidamente a dichos personajes y los ubican en el centro del interés de un público diversificado.

Presentamos los resultados de un trabajo de campo referido al análisis de la recepción por parte de un público culturalmente heterogéneo en el Teatro Piccolo de Milán. Las reacciones de dicho público responden a los principios culturales de cada sociedad: estudiantes italianos de lengua 
española, espectadores argentinos y espectadores españoles interpretan de diferente forma los tiempos de la descortesía, los huecos temáticos y el malestar de los personajes. La diversidad de sus reacciones así lo demuestra. Y ello pese a que no se pueda hablar de trabas en la percepción, sino, muy al contrario, de enriquecimiento intercultural. El posible extrañamiento cultural resulta superado a través de la acumulación de elementos retóricos universales allí donde la plurisignificación no lo ha hecho previamente.

We are asking again for the worldwide success of the Claudio Tolcachir theatre works, especially La omisión de la Familia Coleman, a multiple award-winning work, besides representing over 1500 times in just five years in many theatres around the world.

We have studied systematically repeated ruptures of politeness principles suggested by sociolinguistics, also considering the variation of such principles in each of the companies receiving the theatrical event. It is extremely fitting here the Dubatti's concept of "theatrical event", specifically as a work like La omission ... by way of reception, it becomes a living being ontologically given their social moldability.

We consider deep roots in the specific problems linked to a unique and easily recognizable contemporary society, in their anguish, their shortcomings, their frustrations characters and, in many cases, their actions or inactions consistently true. This realistic force, but also hyperrealism, behaviours and linguistic paralinguistic or pragmalinguistic expressions, define clearly such characters and place them in the center of a diversified public interest arise.

We present the results of a field study based on the analysis of the reception by a culturally diverse audience at the Piccolo Teatro of Milan. The different reactions respond to the cultural principles of each society: Italian students of spanish, Argentinean and Spanish viewers interpreted differently times discourtesy, thematic gaps and discomfort of the characters. The diversity of reactions proves it. And despite the fact that you cannot speak of barriers in perception, but, on the contrary, intercultural enrichment. The potential cultural estrangement is overcome through the accumulation of universal rhetorical elements where the multiple meaning not previously did.

Nous nous interrogeons de nouveau sur le succès mondial du théâtre de Claudio Tolcachir, en particulier de La omisión de la familia Coleman, une œuvre plusieurs fois récompensée, et représentée plus de 1500 fois en seulement cinq ans dans une multitude de théâtres du monde entier.

Pour introduire les possibles causes de ce phénomène, nous étudions de façon systématique la rupture réitérée des principes de courtoisie verbale suggérés par la sociolinguistique, en considérant aussi la variation de ces principes dans chacune des sociétés qui accueillent l'événement théâtral. Le concept d'« événement théâtral » du professeur Dubatti s'avère ici très pertinent, dans la mesure où une œuvre telle que La omisión..., par le biais de sa réception, devient un être ontologiquement vivant du fait sa malléabilité sociale.

Nous considérons le profond enracinement dans des problèmes concrets de personnages uniques et reliés à une société contemporaine parfaitement reconnaissable, dans ses angoisses, ses carences, ses frustrations et, si souvent, ses actions ou inactions clairement véridiques. De cette force réaliste, mais aussi de l'hyperréalisme, surgissent des comportements et des expressions linguistiques, pragma-linguistiques et paralinguistiques, qui définissent nettement ces personnages et les situent au centre de l'intérêt d'un public varié.

Nous présentons les résultats d'un travail de terrain relatif à l'analyse de la réception de l'œuvre de la part d'un public culturellement hétérogène, au théâtre Piccolo de Milan. Les réactions de ce public répondent aux principes culturels de chaque société : étudiants italiens de langue espagnole, spectateurs argentins et spectateurs espagnols interprètent de différentes façons les moments de la discourtoisie, les creux thématiques et le malaise des personnages, comme le montre la diversité de leurs réactions, bien qu'on ne puisse parler d'entraves dans la réception, 
mais bien au contraire, d'un enrichissement culturel. La possible étrangeté culturelle est surmontée grâce à l'accumulation d'éléments rhétoriques universels qui apparaissent là où la pluralité de significations ne l'avait pas fait.

\section{ÍNDICE}

Mots-clés: réception théâtrale, théâtre de la Post-dictature, Argentine, Tolcachir Keywords: receiving viewers, Post-dictatorship Theatre, Argentina, Tolcachir

Palabras claves: recepción teatral, teatro de la Posdictadura, Argentina, Tolcachir

\section{AUTOR}

\section{MARCELINO COTILLA VACA}

Liceo Linguistico Marcelline Tommaseo y Università Cattolica del Sacro Cuore de Milán 